\title{
Significant response to the combination of pyrotinib and letrozole in a patient with metastatic HER2-positive and hormone receptor-positive breast cancer: a case report
}

\author{
Jing Li, Zhengrong Shui, Quchang Ouyang \\ Department of Breast Medicine, Hunan Cancer Hospital, the Affiliated Cancer Hospital of Xiangya School of Medicine, Central South University, \\ Changsha, China \\ Correspondence to: Quchang Ouyang. Department of Breast Medicine, Hunan Cancer Hospital, the Affiliated Cancer Hospital of Xiangya School of \\ Medicine, Central South University, Changsha 410013, China. Email: ouyangquchang@hnca.org.cn.
}

\begin{abstract}
Breast cancer is the most commonly diagnosed malignant tumor and the leading cause of cancerrelated death in women worldwide. Previous studies have demonstrated that patients with human epidermal growth factor receptor 2 (HER2)-positive/hormone receptor (HR)-positive metastatic breast cancer can benefit from HER2-targeted therapy. Pyrotinib, an irreversible tyrosine kinase inhibitor (TKI), has been demonstrated to be effective and safe in treating HER2-positive breast cancer patients. Letrozole is an aromatase inhibitor (AI) which has shown better clinical efficacy when combined with HER2 inhibitors in treating patients with HER2-positive and HR-positive breast cancer than has hormonal therapy alone. However, the effect of combination therapy with pyrotinib plus letrozole in HER2-positive/HRpositive metastatic breast cancer patients has not yet been investigated. In this case report, a 57-year-old female patient with HER2-positive/HR-positive breast cancer received modified radical mastectomy and experienced subsequent relapse and metastasis. She was diagnosed with relapsed right breast cancer, a right chest bone mass accompanied by bone destruction, and metastases in the chest wall and both lungs. She was then enrolled in a phase II clinical trial and was treated with pyrotinib plus letrozole, and achieved a durable clinical response. Our case shows that combination therapy with pyrotinib plus letrozole may provide significant clinical benefit for patients with HER2-positive/HR-positive metastatic breast cancer, with tolerable adverse events.
\end{abstract}

Keywords: Breast cancer; HER2 positive; pyrotinib; letrozole; case report

Submitted Aug 13, 2021. Accepted for publication Sep 18, 2021.

doi: 10.21037/apm-21-2522

View this article at: https://dx.doi.org/10.21037/apm-21-2522

\section{Introduction}

Breast cancer is the most commonly diagnosed cancer and the leading cause of cancer-related death worldwide (1). Human epidermal growth factor receptor 2 (HER2)positive breast cancer accounts for about $15-20 \%$ of all cases, is a more aggressive phenotype, and confers a worse prognosis. Among HER2-positive breast cancer patients, approximately half are also estrogen receptor (ER) positive and progesterone receptor (PR) positive (2-4). Although all HER2-positive breast cancer patients can benefit from anti-HER2 therapy regardless of hormone receptor (HR) status (5), the standard treatment for HER2-positive/ HR-positive metastatic breast cancer patients is still being examined.

Pyrotinib is an irreversible epidermal growth factor receptor (EGFR)/HER2 dual inhibitor developed for the treatment of HER2-positive breast cancer (6), and its promising antitumor activity and safety profile have been confirmed by several clinical trials $(7,8)$. Pyrotinib combined with capecitabine has been approved for treating HER2-positive advanced or metastatic breast cancer in China (9). Letrozole, an aromatase inhibitor (AI), has been 
A

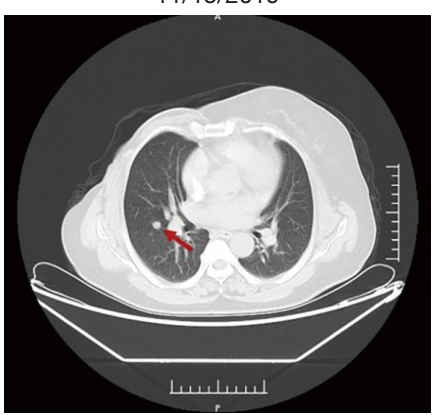

B

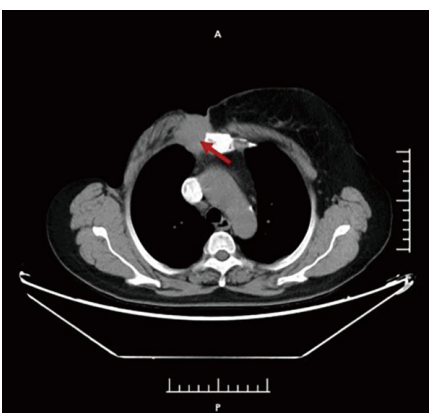

02/25/2020

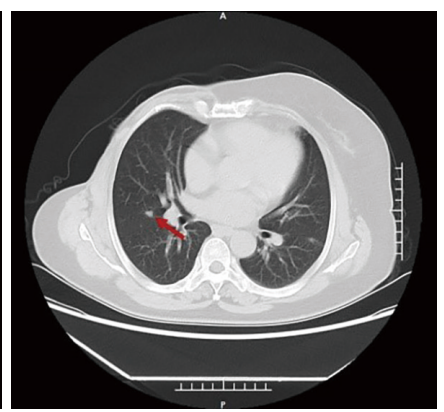

$02 / 25 / 2020$

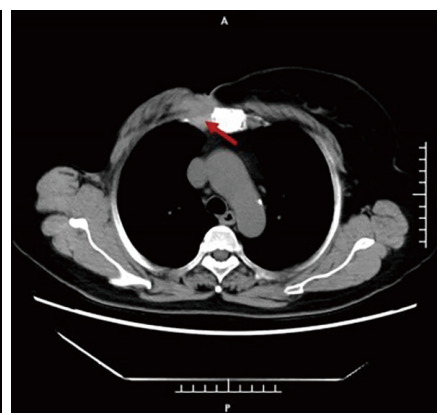

07/13/2020

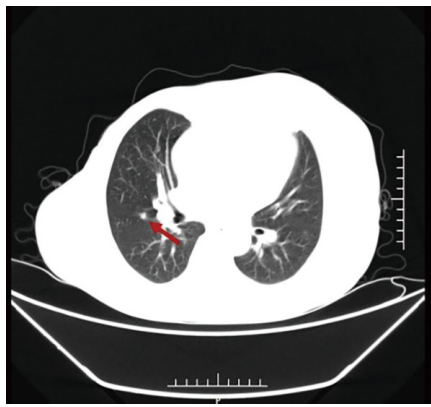

07/13/2020

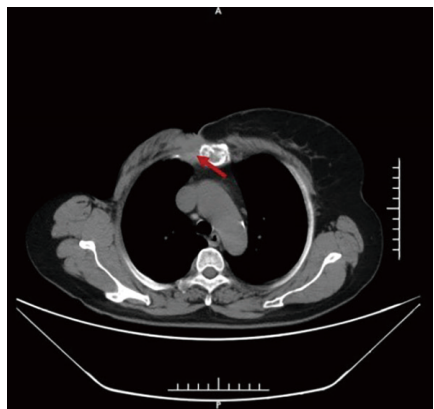

02/24/2021

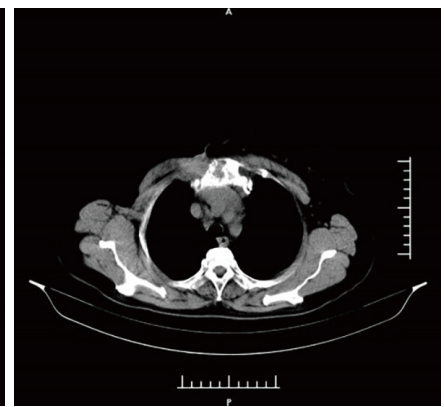

02/24/2021

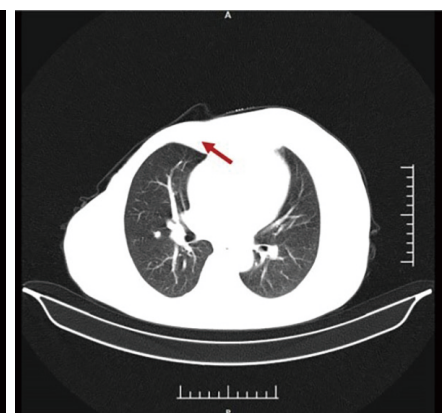

Figure 1 Computed tomography (CT) images of lesions (indicated by arrows): (A) chest bone metastasis; (B) lung metastasis.

proven to have better clinical efficacy when combined with trastuzumab compared with hormonal therapy alone $(10,11)$. Although a similar treatment regimen has been reported previously, the effect and safety profile of pyrotinib plus letrozole in the treatment for HER2-positive/HR-positive patients have not yet been demonstrated (12).

Herein, we report a case of a HER2-positive/HR-positive breast cancer patient who experienced metastasis 2 years after modified radical mastectomy and started combination therapy with pyrotinib plus letrozole. The patient achieved a partial response which was maintained for an extended period, with tolerable adverse events. We present the following article in accordance with the CARE reporting checklist (available at https://dx.doi.org/10.21037/apm-21-2522).

\section{Case presentation}

A 57-year-old postmenopausal woman was diagnosed with right breast cancer and underwent modified radical mastectomy in August 2017. Pathological investigation revealed invasive ductal breast carcinoma (grade II), with immunohistochemistry (IHC) results of ER 3+ (90\%), PR 2+ (60\%), HER2 3+, and Ki-67 3+, (70\%).

In July 2019, the patient found a mass in her right chest wall with diffuse edema and skin redness. As the mass gradually progressed, the patient was admitted to Hunan Cancer Hospital on November 11, 2019. Her Eastern Cooperative Oncology Group (ECOG) performance status (PS) was 0. B-ultrasonography was performed and showed a heterogeneous mass in the medial surgical scar of the right chest wall; hypoechoic nodules in the right supraclavicular area, which were considered to be enlarged lymph nodes; an increased left breast lobule; but no obviously enlarged lymph nodes in both axillary areas and the left supraclavicular area. Computed tomography (CT) revealed a $3.3 \mathrm{~cm} \times 3 \mathrm{~cm}$ mass on the right chest bone with bone destruction, metastases in both lungs with the largest diameter of up to $1 \mathrm{~cm}$, and enlarged mediastinal lymph nodes (Figure 1). On November 15, 2019, local hospital pathological sections revealed c-erbB-2 2+, ER 80\%, PR $80 \%$, and Ki-67 $40 \%$ by IHC (Figure 2). On November 22, 2019, a core needle biopsy was conducted on the chest wall mass and revealed poorly differentiated breast cancerderived carcinoma. which was supported by the medical history of the patient and the IHC results (c-erbB-2 2+, ER $80 \%$, PR 80\%, Ki-67 40\%; Figure 3). Fluorescence in situ hybridization (FISH) identified no HER2 amplification (Figure 4). Based on the above information, the patient was 


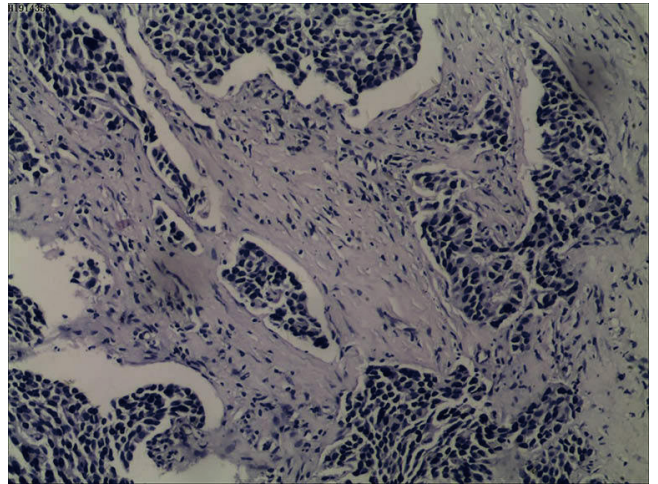

Figure 2 Immunohistochemistry (IHC) showed c-erbB-2 was $2+$, estrogen receptor (ER) was $80 \%$, progesterone receptor (PR) was $80 \%$, and $\mathrm{Ki}-67$ was $40 \%$. The color of tumor cells' nuclei is bluish-purple, and the color of the tumor cell cytoplasm is red $(10 \times 10)$

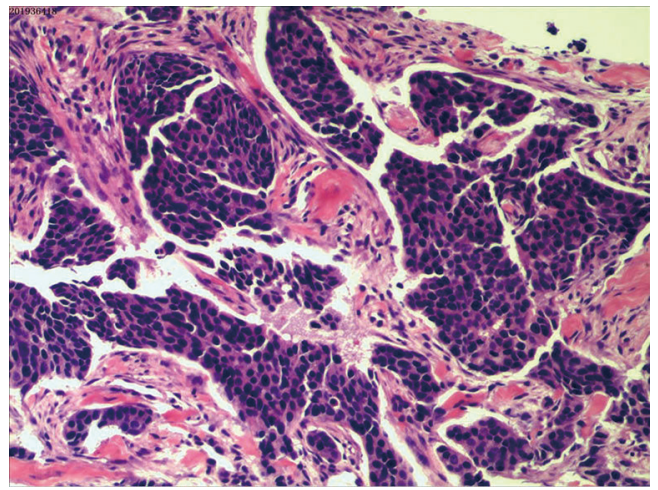

Figure 3 Immunohistochemistry (IHC) of the chest wall mass showed c-erbB-2 was $2+$, estrogen receptor (ER) was $80 \%$, progesterone receptor (PR) was $80 \%$, and $\mathrm{Ki}-67$ was $40 \%$. The color of tumor cells' nuclei is bluish-purple, and the color of the tumor cell cytoplasm is red $(10 \times 10)$.
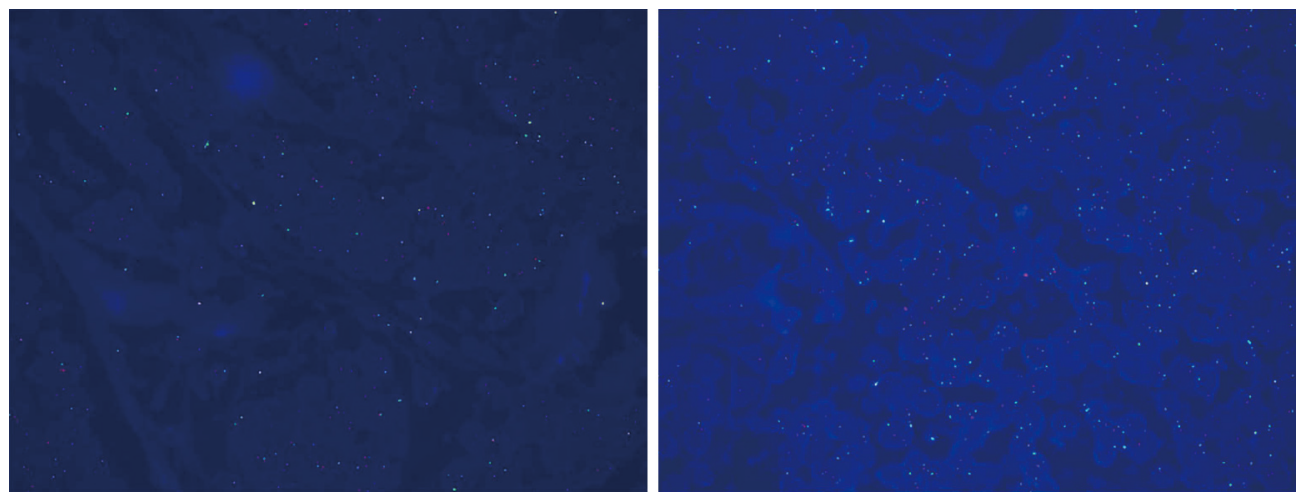

Figure 4 Fluorescence in situ hybridization (FISH) identified no human epidermal growth factor receptor 2 (HER2) amplification (10×100).

diagnosed with relapsed right breast cancer after modified radical mastectomy, right chest bone mass with bone destruction, and metastases in the chest wall and both lungs.

Clinicians suggested that the patient receive trastuzumab plus chemotherapy, but the patient refused this treatment due to financial reasons. She then enrolled in a clinical trial in our hospital entitled "Pyrotinib in Combination With Letrozole in Patients With HER2-Positive, ER-Positive Metastatic Breast Cancer" (NCT04407988) and applied for free treatment.

The patient was then started on pyrotinib alone $(400 \mathrm{mg}$ p.o. daily, every 4 weeks) with letrozole $(2.5 \mathrm{mg}$ p.o. daily, every 4 weeks) from December 3, 2019. On January 29, 2020, remarkable shrinkage of right lung metastatic lesions was confirmed by CT and was evaluated as a partial response (Figure 5). The regimen was discontinued from February 2,
2020, to February 11, 2020 due to the COVID-19 pandemic. The most common adverse event was mild diarrhea, which could be controlled by symptomatic treatment.

At the time of writing, the patient is still alive and is maintaining partial response (Figure 5). All procedures performed in studies involving human participants were in accordance with the ethical standards of the institutional and/or national research committee(s) and with the Helsinki Declaration (as revised in 2013). Written informed consent was obtained from the patient for publication of this case report and accompanying images. A copy of the written consent is available for review by the editorial office of this journal.

\section{Discussion and conclusions}

In the present case, the patient was diagnosed with HER2- 


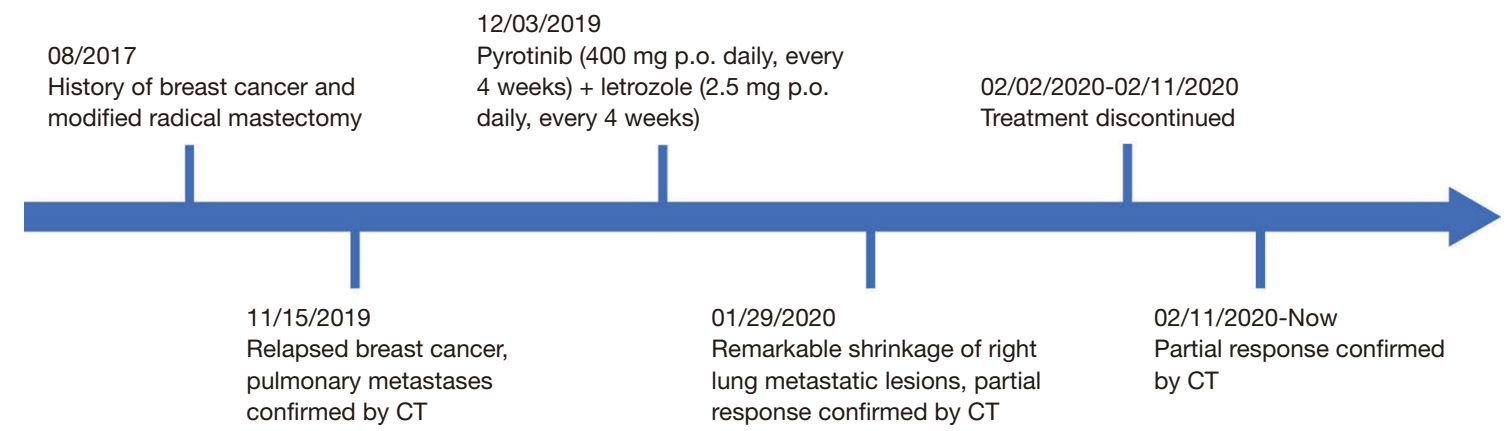

Figure 5 Timeline of diagnosis and treatment. CT, computed tomography.

positive/HR-positive breast cancer and did not receive any adjuvant treatment after modified radical mastectomy, leading to chest bone and pulmonary metastasis after 2 years. The IHC results of the primary tumor were ER $3+(90 \%)$, PR 2+ (60\%), HER2 3+, and Ki-67 + (10\%), indicating sensitivity to anti-HER2 agents and endocrine treatment. However, the IHC results of the metastatic tumor were different from those of the primary tumor, as the metastatic tumor was HER2 negative and HR positive as confirmed by FISH.

According to the fifth European School of OncologyEuropean Society for Medical Oncology (ESO-ESMO) international consensus guidelines for advanced breast cancer (ABC 5), if biological markers of metastatic lesions differ from those of the primary tumor, it remains unknown which result should be used for treatment decision-making (13). The Herceptin adjuvant (HERA) trial demonstrated that 1 year of adjuvant trastuzumab after chemotherapy could significantly improve long-term disease-free survival (DFS) for HER2-positive breast cancer patients compared with the observation group. Furthermore, pertuzumab added to standard adjuvant chemotherapy plus 1 year of treatment with trastuzumab significantly improved the rates of DFS among patients with HER2-positive operable breast cancer in the APHINITY trial $(14,15)$. Therefore, 1-year sequential trastuzumab treatment has become the standard treatment for HER2-positive breast cancer patients irrespective of HR status.

For postmenopausal women with HR-positive breast cancer, endocrine therapy is the mainstay treatment. The EGF30008 trial suggested that for metastatic HER2-positive/HR-positive breast cancer patients, a combined targeted therapy approach is superior to that of endocrine therapy alone (10). The ALTERNATIVE trial demonstrated that lapatinib (LAP) plus an AI could provide superior progression-free survival (PFS) benefit when treating ER and/or PR and HER2-positive metastatic breast cancer patients compared with that of AI alone (16). Therefore, combinations of endocrine therapy plus antiHER2 drugs are currently endorsed by guidelines for treating HER2-positive/HR-positive breast cancer patients $(17,18)$.

Based on the 2020 Chinese Society of Clinical Oncology breast cancer guidelines, HER2 inhibitors combined with chemotherapy has been recommended in treating patients with HER2-positive/HR-positive breast cancer; however, HER2 inhibitors combined with endocrine therapy should only be considered if the patient cannot tolerate the side effects of chemotherapy or has slow progression. In our case, the patient had received 6 cycles of chemotherapy followed by 4 years of toremifene treatment and eventually experienced recurrence, thus meeting the inclusion criteria for the combination treatment with pyrotinib plus letrozole in this patient (19).

Based on the above considerations and the patient's choice, she was recruited to a clinical trial and achieved durable clinical response to combination therapy with pyrotinib plus letrozole. This case suggests that patients with metastatic HER2-positive/HR-positive breast cancer may benefit from combined treatment with pyrotinib plus letrozole, and we recommend clinicians administer targeted therapy such as anti-HER2 or endocrine treatment when at least 1 biopsy result is HER2 or HR positive. We also recommend combination therapy with HER-2 inhibitors plus endocrine treatment to be considered as a maintenance treatment for patients with HER2-positive/ HR-positive breast cancer who achieve stable disease after receiving combination therapy with HER-2 inhibitors plus chemotherapy. Given this is a single case, we are still waiting for further results from the ongoing clinical trial to 
validate this finding.

\section{Acknowledgments}

Funding: None.

\section{Footnote}

Reporting Checklist: The authors have completed the CARE reporting checklist. Available at https://dx.doi. org/10.21037/apm-21-2522

Conflicts of Interest: All authors have completed the ICMJE uniform disclosure form (available at https://dx.doi. org/10.21037/apm-21-2522). The authors have no conflicts of interest to declare.

Ethical Statement: The authors are accountable for all aspects of the work in ensuring that questions related to the accuracy or integrity of any part of the work are appropriately investigated and resolved. All procedures performed in studies involving human participants were in accordance with the ethical standards of the institutional and/or national research committee(s) and with the tenets of the Helsinki Declaration (as revised in 2013). Written informed consent was obtained from the patient for publication of this case report and accompanying images. A copy of the written consent is available for review by the editorial office of this journal.

Open Access Statement: This is an Open Access article distributed in accordance with the Creative Commons Attribution-NonCommercial-NoDerivs 4.0 International License (CC BY-NC-ND 4.0), which permits the noncommercial replication and distribution of the article with the strict proviso that no changes or edits are made and the original work is properly cited (including links to both the formal publication through the relevant DOI and the license). See: https://creativecommons.org/licenses/by-nc-nd/4.0/.

\section{References}

1. National Institutes of Health; National Cancer Institute. Surveillance, Epidemiology, and End Results Program. Cancer stat facts: female breast cancer. Available online: https://seer.cancer.gov/statfacts/html/breast.html

2. Harbeck N, Penault-Llorca F, Cortes J, et al. Breast cancer. Nat Rev Dis Primers 2019;5:66.
3. Loibl S, Gianni L. HER2-positive breast cancer. Lancet 2017;389:2415-29.

4. Slamon DJ, Godolphin W, Jones LA, et al. Studies of the HER-2/neu proto-oncogene in human breast and ovarian cancer. Science 1989;244:707-12.

5. Pogue-Geile KL, Kim C, Jeong JH, et al. Predicting degree of benefit from adjuvant trastuzumab in NSABP trial B-31. J Natl Cancer Inst 2013;105:1782-8.

6. Zhu Y, Li L, Zhang G, et al. Metabolic characterization of pyrotinib in humans by ultra-performance liquid chromatography/quadrupole time-of-flight mass spectrometry. J Chromatogr B Analyt Technol Biomed Life Sci 2016;1033-1034:117-27.

7. Ma F, Ouyang Q, Li W, et al. Pyrotinib or Lapatinib Combined With Capecitabine in HER2-Positive Metastatic Breast Cancer With Prior Taxanes, Anthracyclines, and/or Trastuzumab: A Randomized, Phase II Study. J Clin Oncol 2019;37:2610-9.

8. He L, Zhang F, Ma Y, et al. Pathological Complete Response from Pyrotinib Combined with Trastuzumab, Paclitaxel and Cisplatin in a Postpartum Woman with HER2-Positive Locally Advanced Breast Cancer: A Case Report. Onco Targets Ther 2020;13:8749-56.

9. Blair HA. Pyrotinib: First Global Approval. Drugs 2018;78:1751-5.

10. Johnston S, Pippen J Jr, Pivot X, et al. Lapatinib combined with letrozole versus letrozole and placebo as first-line therapy for postmenopausal hormone receptor-positive metastatic breast cancer. J Clin Oncol 2009;27:5538-46.

11. Rimawi M, Ferrero JM, de la Haba-Rodriguez J, et al. First-Line Trastuzumab Plus an Aromatase Inhibitor, With or Without Pertuzumab, in Human Epidermal Growth Factor Receptor 2-Positive and Hormone ReceptorPositive Metastatic or Locally Advanced Breast Cancer (PERTAIN): A Randomized, Open-Label Phase II Trial. J Clin Oncol 2018;36:2826-35.

12. Wang C, Lin Y, Zhou Y, et al. Pyrotinib with trastuzumab and aromatase inhibitors as first-line treatment for HER2 positive and hormone receptor positive metastatic or locally advanced breast cancer: study protocol of a randomized controlled trial. BMC Cancer 2020;20:653.

13. Cardoso F, Paluch-Shimon S, Senkus E, et al. 5th ESOESMO international consensus guidelines for advanced breast cancer (ABC 5). Ann Oncol 2020;31:1623-49.

14. Cameron D, Piccart-Gebhart MJ, Gelber RD, et al. 11 years' follow-up of trastuzumab after adjuvant chemotherapy in HER2-positive early breast cancer: final analysis of the HERceptin Adjuvant (HERA) trial. Lancet 
2017;389:1195-205.

15. von Minckwitz G, Procter M, de Azambuja E, et al. Adjuvant Pertuzumab and Trastuzumab in Early HER2Positive Breast Cancer. N Engl J Med 2017;377:122-31.

16. Johnston SRD, Hegg R, Im SA, et al. Phase III, Randomized Study of Dual Human Epidermal Growth Factor Receptor 2 (HER2) Blockade With Lapatinib Plus Trastuzumab in Combination With an Aromatase Inhibitor in Postmenopausal Women With HER2Positive, Hormone Receptor-Positive Metastatic Breast Cancer: Updated Results of ALTERNATIVE. J Clin Oncol 2021;39:79-89.

Cite this article as: Li J, Shui Z, Ouyang Q. Significant response to the combination of pyrotinib and letrozole in a patient with metastatic HER2-positive and hormone receptor-positive breast cancer: a case report. Ann Palliat Med 2021;10(9):10124-10129. doi: 10.21037/apm-21-2522
17. Cesca MG, Vian L, Cristóvão-Ferreira S, et al. HER2positive advanced breast cancer treatment in 2020. Cancer Treat Rev 2020;88:102033.

18. Dieci MV, Guarneri V. Should triple-positive breast cancer be recognized as a distinct subtype? Expert Rev Anticancer Ther 2020;20:1011-4.

19. Li J, Jiang Z. CSCO BC guideline: updates for HER2 positive breast cancer in 2020. Transl Breast Cancer Res 2020;1:4.

(English Language Editor: C. Betlzar) 